\title{
Influencia del estilo de vida en personas con alto riesgo genético de enfermedad cardiovascular
}

\author{
Influence of lifestyle in people with high genetic risk for cardiovascular disease
}

\author{
Objetivos \\ Determinar la influencia de un estilo de vida saludable en per- \\ sonas con alto riesgo genético de enfermedad cardiovascular.
}

\section{Diseño y población}

Análisis de tres cohortes prospectivas: 1) The aterosclerosis risk in comunities (ARIC): Personas de etnia blanca y negra de 45 a 64 años de edad ( $n=7.815)$; 2) The women's genome health study (WGHS): Mujeres profesionales de la salud ( $n=21.222) ; 3$ ) The Malmo diet and cancer study (MDCS): 44 a 73 años de edad $(n=22.389)$; y un estudio transversal: 4$)$ The bio image study (BIS): participantes asintomáticos de 55 a 80 años $(n=4.260)$.

\section{Exposición}

Los participantes fueron divididos en quintilos de riesgo cardio-
Khera A y col. N Engl J Med., 2016;375(24):2349-58. vascular basal de acuerdo al polimorfismo genético presente, de los 50 posibles. A su vez, cada persona fue también categorizada de acuerdo a su índice de masa corporal, su estatus tabáquico, su nivel de actividad física y su alimentación (alimentos pobres en grasas, ricos en pescado, pollo y frutos secos).

\section{Resultados}

Los resultados fueron muerte por causa coronaria, infarto agudo de miocardio (IAM) y revascularización coronaria; y para el estudio de corte transversal, calcificación de la arteria coronaria. Los autores analizaron la asociación entre el perfil genético y la incidencia de eventos cardiovasculares ajustando por edad, sexo, nivel educativo y también por el uso de aspirina y vitamina E (solo en el estudio WGHS). La tasa de incidencia de eventos a diez años fue calculada a través de una regresión de Cox.

Tabla 1. Resultados combinados de la influencia del estilo de vida sobre la incidencia de eventos cardiovasculares en pacientes con un perfil genético de alto riesgo cardiovascular.

\begin{tabular}{c|c|c}
\multicolumn{1}{c|}{ Perfil genético } & Estilo de vida & $\begin{array}{c}\text { HR ajustado (IC 95\%) de la combinación de las tres } \\
\text { cohortes }\end{array}$ \\
\hline Bajo riesgo cardiovascular & Favorable & 1 \\
\hline Alto riesgo cardiovascular & Favorable & $1,9(1,62$ a 2,23$)$ \\
& Intermedio & $2,24(1,93$ a 2,61$)$ \\
\cline { 2 - 3 } & Desfavorable & $3,5(2,97$ a 4,12) \\
\cline { 2 - 3 } & &
\end{tabular}

HR ajustado: Hazard ratio ajustado por edad, sexo, nivel educativo, por los cinco componentes de la ascendencia genética, y también por el uso de aspirina y vitamina $\mathrm{E}$ (solo en el estudio WGHS). IC 95\%: intervalo de confianza del $95 \%$.

Entre las personas con alto riesgo genético, un estilo de vida favorable se asoció a una menor calcificación de la arteria coronaria (puntaje 28; IC 95\% 25 a 31) que un estilo desfavorable (puntaje 46; IC $95 \% 40$ a 53).

\section{Conclusión}

En las personas con un perfil genético de alto riesgo, un estilo de vida saludable se asoció con un menor riesgo de desarrollo de eventos y a una menor calcificación de la arteria coronaria.

Fuentes de Financiamiento: ARIC y WGHS: Instituto nacional del Cáncer e Instituto Nacional del Corazón, Pulmón y Sangre. MDCS: Sociedad Sueca del Cáncer, Consejo Sueco de Investigación Médica. Asociación Sueca de Productos Lácteos. Fundaciones Albert Pahlsson y Gunnarnilson. Consejo de la Ciudad de Malmo. BIS: BG Medicina, Abbott Vascular, Astrazeneca, Merck y co.

\section{Comentario}

Las cohortes estudiadas confirman que tanto el perfil genético ${ }^{1,2}$ como el estilo de vida ${ }^{3,4}$ se comportan como predictores independientes del desarrollo de enfermedad cardiovascular.

Sin embargo, vale destacar que no contamos aún con un método indirecto que haya demostrado ser costo-efectivo para evaluar el riesgo genético ya que si bien la presencia de un antecedente familiar de muerte coronaria antes de los 60 años tiene cierta capacidad predictiva, no permite remplazar a las determinaciones genéticas ${ }^{5}$.

Respecto del grado de calcificación de las arterias coronarias, a pesar que se constataron diferencias estadísticamente significativas, en el subgrupo de riesgo cardiovascular intermedio, estas diferencias en la calcificación observadas no tienen la suficiente capacidad predictiva como para reclasificar ("mover" al paciente de la categoría de riesgo intermedio a la de bajo o de alto riesgo), y por lo tanto para cambiar las conductas terapéuticas ${ }^{6}$.

Por otro lado, no hemos identificado trabajos que hayan evaluado el impacto clínico de brindarle al paciente respecto de su adherencia a un estilo de vida saludable.

Conclusiones de la comentadora

Por el momento, tenemos buenos argumentos para seguir recomendando la adopción de un estilo de vida saludable sin que contemos con evidencia para realizar pesquisas de información genómica.

María Florencia Herrera Bornes [ Servicio de Medicina Familiar y Comunitaria del Hospital Italiano de Buenos Aires florencia.herrera@ hospitalitaliano.org.ar ] Herrera Bornes M. Influencia del estilo de vida en personas con alto riesgo genético de enfermedad cardiovascular. Evid Act Pract Ambul. 2017.20(3) JulSep.74. Comentado de: Khera A y col. Genetic Risk, Adherence to a Healthy Lifestyle, and Coronary Disease. N Engl J Med. 2016;375(24):23492358. PMID27959714.

\section{Referencias}

1. Nikpay M y col. A comprehensive 1,000 Genomes-based genome-wide association meta-analysis of coronary artery disease. Nat Genet 2015;47:1121-30.

2. Ripatti S y col. A multilocus genetic risk score for coronary heart disease: case-control and prospective cohort analyses. Lancet 2010; 376: 1393-400.

3. Chomistek A y col. Healthy lifestyle in the primordial prevention of cardiovascular disease among young women. J Am Coll Cardiol 2015; 65:43-51.

4. Akesson A y col. Low-risk diet and lifestyle habits in the primary prevention of myocardial infarction in men: a population-based prospective cohort study. J Am CollCardiol2014; 64: 1299-306.

5. Tada $\mathrm{H}$ y col. Risk prediction by genetic risk scores for coronary heart disease is independent of self-reported family history. Eur Heart J 2016; $37: 561-7$.

6 . Ayoub C y col. Prognostic value of segment involvement score compared to other measures of coronary atherosclerosis by computed tomography: A systematic review and meta-analysis. J Cardiovasc Comput Tomogr. 2017 julio-agosto; 11(4): 258-267. 